\title{
El proceso de escritura y publicación de un artículo científico
}

\author{
Writing and Publishing a Scientific Research Paper
}

\author{
Santos López Leyva' \\ Facultad de Economía y Relaciones Internacionales de la \\ Universidad Autónoma de Baja California, \\ México, campus Tijuana \\ slleyva@uabc.edu.mx
}

Recibido 19 de noviembre de 2012 • Corregido 26 de febrero de 2013 • Aceptado 13 de marzo de 2013

Resumen. El presente trabajo deriva de una experiencia de acción didáctica. Constituye material de apoyo elaborado para un curso de investigación, en una universidad mexicana, en cuanto a la elaboración de artículos científicos. Tiene como propósitos resaltar la importancia del artículo científico como mecanismo de difusión de la ciencia y explicar los pasos para su publicación en una revista. Define el concepto de artículo científico y analiza su estructura. Revisa componentes, separándolos en centrales y conexos. Los primeros son: introducción, métodos, resultados, discusión y conclusiones. El título, resumen, palabras claves, agradecimientos, referencias y anexos se denominan partes conexas. Ofrece sugerencias para la elaboración de cada una de estas secciones. Formula recomendaciones para la preparación del manuscrito y sugiere las etapas que debe tener el proceso de publicación. Con base en entrevistas a seis investigadores, marca las restricciones, y propone recomendaciones para la escritura y publicación de un artículo.

Palabras claves. Artículo científico, arbitraje científico, difusión científica, producción científica, publicaciones seriadas, Revista Electrónica Educare, Costa Rica.

Abstract. This paper summarizes a didactic experience. It has been used in a research course taught in a Mexican university for the preparation of scientific papers. It highlights the importance of scientific papers as dissemination channels for science and describes the steps for publishing in specialized journals. The concept and structure of scientific papers are explained. This study includes the analysis of the main sections in a scientific paper: introduction, methods, results, discussion, and conclusions, as well as the connecting sections: title, abstract, key words, acknowledgments, references, and annexes. It provides recommendations on writing these sections, preparing the manuscript, and the steps to accomplish in the publication process. In addition, based on interviews to six researchers, this paper points out limitations and provides recommendations on writing and publishing a scientific paper.

Keywords. Scientific paper, review, scientific diffusion, scientific production, scientific publications, serials journals, Educare Electronic Journal, Costa Rica.

1 Es profesor de tiempo completo de la Facultad de Economía y Relaciones Internacionales de la Universidad Autónoma de Baja California, México, campus Tijuana. Realizó sus estudios de Doctorado en la División de Posgrado de la Facultad de Economía de la Universidad Nacional Autónoma de México (UNAM), en el área de Economía de la Ciencia y la Tecnología. Pertenece al Sistema Nacional de Investigadores (SNI) desde 1998, Nivel II. Es responsable del cuerpo académico "Estudios del Desarrollo Global". En docencia imparte las cátedras de Economía mundial, Economía de la educación e Investigación dirigida en licenciatura y posgrado. 
URL: http://www.una.ac.cr/educare

CORREO: educare@una.cr

\section{Introducción}

El presente trabajo tiene como propósito presentar los elementos analíticos y técnicos necesarios que posibilitan la escritura y publicación de un artículo científico.

El artículo científico es la principal forma de comunicación de la ciencia; no es la única, pero es la mayormente valorada: experimenta un proceso de revisión por pares, quienes -al aprobar el trabajo- constatan su buena calidad y verifican que atienda los elementos necesarios para fortalecer el conocimiento científico en la temática en cuestión. En este aspecto, es necesario tener en cuenta opiniones de algunos campos disciplinarios, tal es el caso de las ciencias sociales, donde tienen alto valor los capítulos en libros, aunque difícilmente desarrollan el mismo proceso de revisión y evaluación que un artículo científico.

Para la realización de esta publicación, se recurrió a la consulta de una amplia fuente de recursos bibliográficos; también se contó con el apoyo de seis investigadores, miembros del Sistema Nacional de Investigadores nivel III en México -el cual constituye el máximo grado que establece este Sistema-, de quienes se recogieron importantes aportaciones a través de la aplicación de un cuestionario; además, dos de ellos colaboraron en forma directa para el mejoramiento de la presente comunicación².

El trabajo se divide en ocho apartados, el primero resalta la importancia de los artículos y las revistas científicas como principal forma de comunicación de la ciencia; el segundo se refiere a la definición y estructura del artículo científico; después se atienden las denominadas partes centrales del artículo y, enseguida, lo que se conoce como partes conexas; en quinto lugar se aborda el proceso de preparación del manuscrito; después el proceso de publicación; en el apartado siguiente se apuntan algunas restricciones y recomendaciones, después se recoge la opinión de los investigadores en torno a su experiencia como evaluadores de artículos científicos en revistas indexadas y, por último, se ofrecen las conclusiones.

\section{El artículo científico como forma de comunicación de la ciencia}

La principal forma de comunicar la ciencia entre las comunidades científicas es a través de los artículos científicos. La publicación de un artículo como resultado de una investigación es parte del proceso mismo de la producción científica, por tanto, una investigación no se termina hasta que sus resultados sean publicados (Mantilla-Villarreal et al., 2010; Mari-Mutt, 2010).

Diferentes autores mencionan una serie de elementos que justifican las ventajas que representa la publicación en revistas científicas sobre los libros y otras formas de difusión académica a las cuales se les denomina literatura gris, prófuga o semipublicada.

2 El autor agradece el apoyo en la revisión de este trabajo a los doctores Eduardo René Fernández González y Óscar Fernando Contreras Montellano.

6

Santos López Leyva 
Entre las razones para preferir la publicación en una revista, sobresalen las siguientes (Cargill y O'Connor, 2009; Rogel, 2009; Sabaj, 2009):

Mediante esta vía: a) las investigaciones están en proceso y sujetas a discusión de los diferentes grupos de científicos; $b$ ) los artículos y revistas pasan por un proceso de revisión por pares, quienes se encargan de evaluar la calidad científica de las publicaciones; c) es posible generar indicadores de posicionamiento al interior de una comunidad académica, ya que una revista aparece en forma periódica; d) se establecen comparaciones en el contexto mundial, dado que se dispone de estándares internacionales, tanto académicos como de formato para la producción de revistas y elaboración de artículos, para ello se encuentran diferentes bases de datos que evalúan y registran las publicaciones con lo que aumentan su visibilidad.

Además de lo anterior, la creación científica, manifiesta en revistas y artículos, hace posible disponer de una existencia de producción original importante y actualizada de conocimiento; mejora el acervo mediante el intercambio; se tiene la posibilidad de acceder a literatura internacional reconocida; fomenta una mayor autonomía para la comunidad de científicos, tanto en el contexto local como internacional; mediante la publicación de artículos y revistas se incrementa la visibilidad del conocimiento; las revistas funcionan como un medio de comunicación e información entre las comunidades científicas; al impulsar la evaluación actúan como mecanismos de entrenamiento para árbitros, autores y editores; constituyen un testimonio documental de la creación científica; conforman un medio eficaz para la enseñanza de la ciencia; integran un instrumento para definir la política científica de un país o de una institución, y apoyan en la promoción de un campo disciplinar.

Contreras (2011) establece que: "No se trata de medios de difusión masiva, ni de fuentes de divulgación político-ideológica, ni siquiera de plataformas para promover soluciones a los problemas técnicos o sociales, sino de espacios regidos por normas de argumentación y validación del conocimiento especializado que las propias comunidades aceptan como legítimas, destinados a publicar resultados de investigaciones académicas y dirigidos en principio a un público de pares, no al público en general". (p. 12)

En opinión de los investigadores entrevistados, cuando se lee un artículo en una revista arbitrada, se puede estar seguro de que la información manejada ha sido validada por pares, y se encuentra en la frontera del conocimiento; además, el prestigio de la revista se trasfiere implícitamente al artículo. Las revistas imponen criterios de exigencias de calidad, creatividad, objetividad, seguridad -ya que el artículo es evaluado por pares-, posibilidad de recibir retroalimentación y sugerencias para mejorar el escrito. Esto se aplica, por supuesto, solo a las revistas de alto nivel. La aportación es original y queda en forma escrita. Nos dice el doctor S. Álvarez (comunicación personal, 19 de junio, 2012) que: "el artículo debe ser principalmente en inglés para asegurar una mayor audiencia. Nos guste o no, el inglés es la lengua más universal 
URL: http://www.una.ac.cr/educare

CORREO: educare@una.cr

del momento, como alguna vez lo fue el latín". Es necesario agregar que en la actualidad existen diversos bancos de información científica encargados de reconocer múltiples publicaciones científicas en español.

Por otra parte, es posible desarrollar indicadores de producción científica de cada país y llevar a cabo una comparación de las revistas sin importar el área de conocimiento, ni el tamaño de estas. Los principales indicadores son el factor de impacto, el índice de inmediatez, el índice de media vida y el índice de Hirsh. El factor de impacto se obtiene de una razón del número de citas que reciben los artículos en los dos años siguientes de su publicación entre el número de artículos que publica la revista; el índice de inmediatez es el número de citas que recibe en el siguiente año de su publicación, entre el número de artículos que publica la revista, y el índice de vida media se refiere al periodo en que la revista recibe el $50 \%$ de las citas.

Aunque el factor de impacto es el mayormente usado, este ha recibido serias críticas, aparte de las realizadas por el propio autor Garfield (2003) y otras que provienen de: 1) la calidad del artículo no puede estar limitada por el tiempo; 2) el número de revistas contenidas en la base de datos del ISI (Institute for Scientific Information) es una proporción pequeña de todas las revistas científicas; 3 ) las revisiones son citadas con mayor frecuencia que la investigación original; 4) no toma en cuenta las autocitas, las cuales constituyen la tercera parte de todas las citaciones; 5) los errores son comunes en las listas de referencia, se ha encontrado que aproximadamente alcanzan el $25 \%$; 6) no toma en cuenta los ambientes y condiciones sociales de cada país, y 7) está muy cargado hacia la medición de la ciencia producida en los Estados Unidos (Cortex, 2001; Licea y Santillán-Rivero, 2002; Quispe, 2004). Garfield (2003) señala que este indicador no se construyó para medir la productividad de los investigadores, lo cual constituye una desviación en su uso; además está sesgado hacia el idioma inglés y hacia las investigaciones en ciencias médicas.

Aunque el artículo científico no es la única vía de comunicación de la ciencia, si constituye la forma más valorada en la actualidad, debido a que todas las revistas científicas disponen de un sistema de revisión por pares, cuyo propósito es evaluar y filtrar las diferentes contribuciones que reciben.

El artículo científico es un texto que destaca los resultados de un proyecto de investigación académica, concluida o en proceso, que posterior a una rigurosa revisión por especialistas es considerado como una contribución original y relevante para el desarrollo de un campo del conocimiento científico. Este debe mostrar las siguiente características: a) tener como base los resultados de una investigación científica original y relevante; $b$ ) abarcar parte del estado del conocimiento vigente; c) estar escrito con lenguaje adecuado, y reunir una serie de condiciones formales y de contenidos propias de un texto científico; d) ser respaldado por una institución que auspicie la investigación; e) la pertinencia de los textos, en todos los casos, deberá estar determinada por expertos en la materia (López-Leyva, Alvarado-Borrego y Mungaray-Moctezuma, 2011; Rogel, 2009; Sabaj, 2009). 
La originalidad es una premisa del artículo científico, otra es la claridad para comunicar las ideas; además, debe contar con una buena organización, es decir, el ordenamiento que siguen las partes que conforman la comunicación debe ser adecuado. Lodeiros-Seijo, De Donato y Monge-Nájera (2002) establecen que un buen artículo científico debe poner en práctica tres principios básicos de redacción: a) precisión, uso palabras que comuniquen con exactitud lo que se quiere decir; b) claridad, el texto se debe entender con facilidad, y c) brevedad, debe incluir solo la información necesaria para comunicar lo que se desea. Según Baltzopoulos (2003), existen cuatro elementos por tener en cuenta en la escritura de un artículo científico: relevancia y claridad; originalidad; datos y métodos adecuados, y un buen control experimental y su correspondiente análisis estadístico. Otro elemento al que es necesario brindar mucha atención en la escritura de un artículo científico es la articulación, a la cual González y Jiménez (2012, p. 6) definen como: “(...) una lógica de construcción donde existe correspondencia entre los conceptos y planteamientos que dan lugar a las preguntas de investigación y a la metodología para encontrar información a fin de darles respuesta (...)".

\section{La estructura del artículo científico}

Las partes que conforman un artículo científico son: título, autores, resumen o abstract, palabras claves (keywords), introducción, objetivos, métodos, resultados, discusión, conclusiones, agradecimientos, referencias, anexos, tablas y figuras (Campanario, 2003; López-Leyva et al. 2011; Sharp, 2002).

Las partes del artículo se pueden dividir en centrales y conexas (López-Leyva et al., 2011). Se incluyen como partes centrales de un artículo: introducción, métodos, resultados, discusión y conclusiones. Las restantes se consideran como partes conexas (Cargill y O'Connor, 2009).

En la elaboración de un artículo se requiere, en primer lugar, disponer de buena información, exponer datos novedosos y bien trabajados. La presentación y discusión de resultados se convierte en el principal referente de una comunicación científica de este tipo. Disponiendo de buenos resultados y de una adecuada interpretación de estos, el paso siguiente es la elaboración de la introducción, donde se trata el problema en cuestión, después se le ofrece una dirección al problema en la definición y delimitación de variables hacia aspectos más específicos; por último, se conecta la introducción con la información que se ha trabajado. En algunos casos, principalmente en las ciencias sociales, se recomienda elaborar una introducción pequeña que solo explique el problema y después se construye un buen marco teórico en el cual se sustenten las siguientes partes del artículo. Luego se pasa a la definición y explicación del apartado de métodos, los cuales se encargan de ofrecer mayor credibilidad a los resultados ya que se expone cómo estos fueron obtenidos y la forma en que se trabajaron. Por último, la discusión, la cual empieza con el análisis de los resultados y termina con la comparación o la revisión de los elementos que fueron planteados en la introducción. A esta estructura del artículo se le ha denominado AIMRaD (Abstract, introducción, materiales y métodos, resultados y discusión) (Cargill y O'Connor, 2009; Sharp, 2002). Estos autores 
URL: http://www.una.ac.cr/educare

CORREO: educare@una.cr

también consignan la forma AIRDaM (Abstract, introducción, resultados, discusión y materiales y métodos), donde al final se explica cómo se llevó a cabo la investigación. Otra variante para presentar el artículo es AIM(Rad)C (Abstract, introducción, materiales y métodos, discusión de resultados por cada variable y conclusiones). Esto último consiste en discutir, en forma separada, los resultados de cada variable; entonces habría tantas discusiones de resultados como variables explicativas tenga la investigación.

\section{Las partes centrales del artículo}

A continuación se realizará un análisis de las componentes principales del artículo científico.

\section{Resumen (Abstract)}

Esta sección debe convencer al lector de la importancia del artículo y atraerlo a su lectura completa, pero también se debe considerar que los organismos que elaboran bancos de información científica utilizan dicho apartado para hacer la clasificación del artículo. Cada revista fija el número de palabras o renglones que debe tener la extensión del resumen. En dicho espacio es preciso incluir las principales ideas que se atienden en las partes centrales del artículo.

Day y Gastel (2006) sugieren que el resumen debe ser el artículo en pequeño. En tanto que Mantilla-Villarreal et al. (2010) sostienen que esta parte es una versión compacta y condensada del artículo, es decir, una presentación del AIMRaD en un número reducido de palabras, debe contener: objetivos y alcances de la investigación, métodos empleados, resultados, discusión y conclusiones.

Según Belcher (2009), el resumen no debe ser solo el encuadre del tópico que trata la investigación, no es el plan de trabajo del artículo, no es un bombardeo de resultados, no incluir notas de pie o aclaratorias, no incluir citas ni parafrasear autores, y no incluir abreviaturas, símbolos, acrónimos ni fórmulas matemáticas. El resumen es la palabra del autor.

\section{Introducción}

Este apartado debe contener: a) declaraciones acerca del campo de investigación; b) declaraciones más específicas acerca de las partes que no han sido investigadas y ofrecer fundamentos de investigación aún desconocidos; c) elementos que indican la necesidad de contar con un conocimiento mayor sobre la problemática; d) componentes que ofrezcan los alcances, propósitos y objetivos de la investigación, y e) elementos que brinden una valoración positiva o una justificación adecuada para llevar a cabo el estudio (Cargill y O'Connor, 2009; Mantilla-Villarreal et al. 2010). 
Day y Gastel (2006) establecen las siguientes reglas para la elaboración de una buena introducción: a) deberá ser escrita en presente, estableciendo, con la mayor claridad posible, la naturaleza y alcances del problema investigado; $b$ ) incluir un breve y pertinente repaso de la bibliografía más relevante del tema; c) establecer los métodos y señalar las razones por las que fueron seleccionados; d) formular los principales resultados de investigación, y e) mencionar conclusiones centrales a las que llega el estudio.

Dice Körner (2004) que la introducción debe tener el suficiente espacio que permita: a) ubicar la investigación en el contexto de los trabajos más relevantes en el tema; b) explicar las razones para la realización del estudio; c) mencionar los métodos utilizados; d) proveer algunos adelantos de la discusión y las conclusiones. También señala que si esta ocupa más de dos terceras partes de los resultados y discusión de los mismos, resulta demasiado larga.

\section{Métodos}

La importancia de esta sección consiste en que soporta la credibilidad de los resultados encontrados, porque en ella se debe señalar cuáles fueron los mecanismos, formas y caminos para su obtención y tratamiento de los datos que permitieron llegar a los resultados obtenidos (López-Leyva, et al., 2011).

Booth (2006) recomienda empezar la escritura del artículo con esta sección, porque presume que es la más fácil de escribir, puesto que se tiene claro lo que se hizo, cómo se hizo y los resultados encontrados.

Belcher (2009) formula una serie de recomendaciones para atender esta sección, las cuales son: identificar en forma muy clara los métodos utilizados, describir con claridad y seguridad el tamaño y características de la muestra e identificar los procedimientos mediante los cuales se obtuvo esta; definir las dimensiones de medida utilizadas para cada una de las variables, así como los instrumentos de medición; aclarar el contexto de la investigación, donde se llevó a cabo esta y las circunstancias que rodearon el proceso; una acertada definición de las variables, cuáles funcionan como dependientes y cuáles como independientes, establecer las formas de relacionarse entre estas y las influencias de unas sobre las otras; escribir esta sección en tiempo pasado, donde se permite utilizar la voz pasiva; no pretender que se convierta en un tratado matemático o de funciones estadísticas; evitar la mezcla con la sección de resultados y la discusión de estos; revisar y atender las instrucciones que ofrece la revista seleccionada. Una de las características de este apartado es su brevedad.

\section{Resultados y discusión}

Cargill y O'Connor (2009) recomiendan que la discusión se encuentre muy relacionada con el título del trabajo. Además, el mayor acercamiento tiene que ser con la introducción; dejar 
URL: http://www.una.ac.cr/educare

CORREO: educare@una.cr

claramente conectados los resultados con cada uno de los puntos que se presentaron en esta. Esta sección debe atender: a) una referencia bien fundamentada al propósito del estudio, a la hipótesis central; b) revisar los resultados en orden de importancia, resaltar si los resultados sustentan en forma adecuada la hipótesis principal del trabajo, contestan las preguntas de investigación y alcanzan los objetivos propuestos; c) las explicaciones de los resultados están soportadas en forma suficiente por la sólida y actualizada bibliografía manejada; $d$ ) establecer las limitaciones del estudio, las cuales evitan la aplicación generalizada de los resultados; e) implicaciones del estudio, tanto desde la perspectiva del contexto del espacio, de tipo referencial, como desde la perspectiva teórica y recomendaciones para futuras investigaciones.

Tanto para la introducción como para esta sección, pero sin duda se debe generalizar a todo el artículo, evitar afirmaciones como: 'un considerable monto de,' 'un considerable número de', 'la mayoría de', 'un pequeño número de', 'es absolutamente esencial', etcétera, en general expresiones carentes de precisión. En este sentido, es muy útil revisar las 213 expresiones de la jerga común presentadas por Day y Gastel (2006), las cuales deben evitarse en los trabajos científicos. También resulta de utilidad atender los 27 puntos que presenta Garson (2002) para la discusión de resultados y las conclusiones de un artículo científico. Aquí se presentan los de mayor observancia: 1) cuidar que esta sección no se convierta en una revisión general de las anteriores; 2) las inferencias se encuentran debidamente relacionadas con la propuesta de investigación; 3) es congruente con la revisión bibliográfica; 4) es congruente con los datos y métodos; 5) atiende las preguntas por orden de importancia; 6) el orden de presentación es el adecuado; 7) ofrece datos apropiados para cada una de las variables; 8 ) no omite información importante; 9) revisar el tamaño de la muestra; 10) se considera el corto y largo plazo; 11) se atienden las condiciones en las cuales son válidos los resultados; 12) se revisan los efectos posibles; 13) las conclusiones se derivan en forma adecuada de las premisas; 14) cuando se usan medidas de tendencia central, se atienden las dispersiones; 15) se tiene cuidado de que los trabajos y documentos con los que se compara el artículo sean de calidad; 16) los expertos que se consultan, realmente lo son y 17) verificar la veracidad, cuando se recurre a testimonios.

\section{Conclusiones}

Se recomienda no confundir la discusión de resultados con las conclusiones, ni repetir textualmente lo dicho en el resumen; las conclusiones dependen de los resultados y de su análisis, toman en consideración tanto el marco teórico como los objetivos; deben obtenerse a partir de algo más que de los datos, y buscan resaltar el principal aporte de la investigación (Rogel, 2009).

Además de las conclusiones derivadas en forma directa de la realización de la investigación, es necesario incluir en esta sección información que delimite la aportación, tal es el caso de las posibles interpretaciones alternativas que se descartan en el trabajo; las potenciales líneas adicionales de investigación a la vista de los resultados obtenidos, y la relevancia de los aportaciones del trabajo de investigación frente a otros trabajos similares. 


\section{Las partes conexas del artículo científico}

\section{Título}

Esta constituye la primera parte del artículo que se encontrarán los lectores y árbitros, de tal manera que debe buscarse un título sugestivo que invite a la lectura del artículo, es lo último que se escribe, pero lo primero en leerse. Debe atraer a los lectores, en particular a la audiencia científica, para que busquen el artículo. Se deben evitar palabras ambiguas, abreviaturas y expresiones tales como: 'algunos aspectos de', 'algunos elementos de', 'investigaciones acerca de', 'estudios preliminares sobre,' 'notas acerca de', 'observaciones sobre,' 'consideraciones acerca de', entre otras. La American Psychological Association (APA) (2010, p. 23) plantea que: "lo largo de un título no debe pasar de 12 palabras" ${ }^{3}$.

\section{Autor o autores}

Como producto de la generalización en el uso de los sistemas de puntaje para evaluar el desempeño académico de los investigadores, en nuestros tiempos se ha incrementado el interés por aparecer en la autoría de una obra académica. La pregunta es quiénes deben aparecer como autores. Se establece que solo deben figurar las personas que hayan hecho un trabajo significativo en la concepción, diseño, análisis e interpretación de los datos; en la escritura y revisión crítica del trabajo relativa a contenidos intelectuales, y aprobación final de la versión que se va a publicar (Campanario, 2003). Cada una de las disciplinas científicas dispone de estilos diferentes para colocar la lista de autores, pero en todos los casos, como apunta Rojas (2012, p. 56) existe "la responsabilidad de dar crédito a los autores o autoras, cuyos textos o ideas se utilizan en el escrito, constituye una obligación ética de consenso en el ámbito académico y social (...)".

En el campo de los autores y las autoras, también se debe incluir su adscripción institucional; algunas revistas solicitan otros datos como el correo postal, teléfono y correo electrónico.

\section{Palabras claves (keywords)}

El número de palabras claves y la forma de seleccionarse son establecidos por la revista. Para la preparación de estas, deben seleccionarse palabras vinculadas al problema de investigación; pueden ser sustantivos compuestos, pero nunca frases; es recomendable seleccionar palabras vinculadas al problema, a los métodos y a los resultados (Rogel, 2009).

Algunas áreas disciplinarias cuentan con tesauros prestablecidos de los cuales se seleccionan las palabras claves, por ejemplo el JEL (Journal of Economic Literature), cuyo

3 Traducción del autor. 
URL: http://www.una.ac.cr/educare

CORREO: educare@una.cr

nombre completo es Journal of Economic Literature Classification System, para el caso de las ciencias económicas; el Medical Subject Headings (MeSH), para el caso de las ciencias médicas. En México se ha establecido el IRESIE (Índice de Revistas de Educación Superior e Investigación Educativa) como un tesauro para el área de la educación superior. En el caso de la investigación agrícola funciona el NALT (National Agricultural Library Thesaurus). Así, en cada caso, los investigadores deben conocer si su disciplina cuenta con el tesauro correspondiente.

\section{Agradecimientos}

Para esta sección se siguen diversas prácticas en función de la disciplina científica de que se trate, pero debe incluir colaboradores técnicos, auxiliares de investigación, personas que ayudaron o apoyaron la investigación, entidades financieras que sufragaron los gastos del proyecto, instituciones que colaboraron y colegas que revisaron las versiones previas (Rogel, 2009). Debe evitarse agradecimientos excesivamente efusivos o familiares, la sección debe ser breve y directa. Day y Gastel (2006) señalan que deben existir dos tipos de agradecimientos. En primer lugar a quienes participaron en la elaboración técnica y revisión del artículo, pero que no alcanzan el mérito para ser reconocidos con la autoría; en segundo lugar, quienes proporcionaron apoyo financiero y administrativo.

\section{Citas}

Se pueden establecer dos aspectos esenciales respecto de las citas: primero su naturaleza textual y, segundo, su función. Con respecto a su naturaleza, la cita constituye un texto que, por definición, es dependiente de otro. Segundo, la cita establece un tipo específico de relación respecto del texto madre (Sabaj y Páez, 2010).

Aunque las citas no constituyen una sección de la estructura del artículo, se introducen en este apartado ya que se les debe ofrecer especial atención porque pueden ser uno de los motivos de rechazo del trabajo. Aunque existen una serie de recomendaciones para el tratamiento de las citas, en este aspecto las revistas son muy precisas y establecen, de manera clara, la forma de citar cada una de las fuentes utilizadas.

\section{Referencias}

Para la presentación de las referencias también es muy importante seguir al pie de la letra las instrucciones de la revista, pero los formatos más comunes son: a) relación alfabética al final del artículo; b) notas de pie de página, y c) relación numérica al final del artículo, en este caso cada referencia aparece precedida de un número (Campanario, 2003). 
En general, en los diferentes formatos aparecen los siguientes datos: apellidos y nombres de los autores, año de publicación, título del artículo o bien la obra consultada, nombre de la revista donde se publicó el trabajo, volumen y número en que se publicó, institución editora, páginas inicial y final.

Comunmente, cuando los estudiantes de posgrado inician su trabajo de investigación, una de las preguntas obligadas es: ¿cuántas referencias debe llevar un trabajo de tesis? Para un reporte de investigación no existe un número mínimo o máximo de referencias, esto depende del tipo de artículo y el soporte teórico y metodológico necesario.

\section{Anexos}

En los anexos deben aparecer: a) los instrumentos utilizados para la obtención de los datos, tales como cuestionarios, entrevistas y encuestas; $b$ ) datos demográficos de la muestra y la población; fórmulas, demostraciones y otro tipo de algoritmos especiales; c) materiales usados, por ejemplo algunos tipos de pruebas utilizadas en psicología; d) archivos originales, archivos históricos; e) programas informáticos, y f) especificaciones técnicas de la muestra (Campanario, 2003). Como resultado de que las revistas son muy exiguas en el espacio, se recomienda que los autores cuenten con una página web donde remitir al lector para la consulta de algunos elementos adicionales del artículo.

\section{Proceso de preparación del manuscrito}

La preparación del manuscrito debe atenderse en cuatro apartados. En primer lugar es necesario definir un plan de escritura, considerando el tiempo y los recursos disponibles; después definir la etapa inicial de preparación, es decir, cómo iniciar el escrito; atender los procedimientos de edición y, por último, llevar a cabo la revisión final.

\section{Definición del plan de escritura}

Una autora recomienda definir un plan de actividades que comprende doce semanas desde el inicio del artículo hasta que se envía a la revista (Belcher, 2009). Durante ese lapso se les debe asignar tiempo a las diferentes actividades, tales como: el diseño del plan y la forma de seguimiento de las actividades; forma de empezar el artículo, discusión del plan de escritura con colegas, escribir las principales ideas del artículo, hacer un borrador de los principales argumentos, seleccionar la revista, se recomienda que se evalúen unas tres revistas para tomar la decisión final; realizar la revisión de las fuentes teóricas, trabajar los datos y las evidencias con las cuales se contrastan las hipótesis. Se recomienda que el artículo quede terminado en la octava semana de trabajo. Las siguientes cuatro semanas se dedicarán a la revisión y edición del trabajo, al final de la décimo segunda 
URL: http://www.una.ac.cr/educare

CORREO: educare@una.cr

semana el artículo se enviará a la revista. Mantilla-Villarreal et al. (2010) recomiendan dividir el trabajo en dos etapas: 1) la planificación, etapa en la que se debe desarrollar las actividades encaminadas a la organización de la investigación, y 2) cuerpo del artículo, donde se muestran los componentes del artículo y los parámetros por tener en cuenta al momento del desarrollo del tema.

\section{Etapa inicial de preparación}

Una de las fases más difíciles es empezar la escritura del artículo. En esa fase, Cargill y O'Connor (2009) recomiendan considerar catorce elementos: 1) seleccionar el agregado de datos que se manejarán en el artículo; 2) hacer reuniones con el equipo o comentar con colegas; 3) revisar la información que proporciona a los colaboradores, en este aspecto es muy importante revisar los diferentes modelos de artículos que acepta la revista; 4) definir la forma de presentar los datos y resultados, cuáles se presentarán y cuáles no, si se utilizarán gráficas y cuadros; 5) remarcar las viñetas o ideas fuerza que contendrá el artículo; 6) sugerir y analizar alternativas para el nombre del trabajo; 7) redactar un borrador de métodos, procedimientos y resultados; 8) 9) elaborar un borrador de la introducción y el marco teórico, 10) preparar un borrador de la discusión de resultados y de las conclusiones, 11) escribir un borrador del abstract, 13) hacer un borrador de palabras clave, y 14) iniciar la escritura del manuscrito organizando los borradores y notas de todas las partes que hasta aquí se han elaborado.

\section{Atender los procedimientos de edición}

Cuando se termine el trabajo, lo primero que se debe hacer es alejarse de él, esto es, guardarlo por alrededor de 48 horas, con la finalidad de refrescar los pensamientos e ideas en torno a su contenido. Después de ese lapso, entrar de nuevo al trabajo y marcar todos los errores posibles, no corregirlos de la primera lectura, sino solamente marcarlos. Siempre es necesario guardar todas las correcciones, no eliminar los borradores, porque es posible que se necesiten en el futuro. Iniciar el proceso de restructuración. El proceso de revisión y restructuración se puede repetir en varias ocasiones, hasta lograr satisfacción con el contenido del trabajo. El siguiente paso es editar, revisar la fluidez del discurso, los significados y estructura de las oraciones, que se manejen ideas completas, que hilvanen las partes del artículo y los párrafos en la escritura. Revisar la ortografía, la puntuación y la gramática; revisar la correspondencia de citas con las referencias, y la consistencia de estas. Revisar los encabezados para ver si corresponden a los contenidos y atienden las especificaciones de la revista, editar las tablas y figuras. Repetir estos procesos cuantas veces sea necesario. 


\section{Un repaso final}

Cuando ya se tiene el artículo completo, incluso ya se haya realizado la edición, es importante llevar a cabo una revisión final de forma integral, con especial atención en: el título, realizar un ejercicio comparando varios títulos, verificar si cuenta con palabras adecuadas que atraigan al lector; en la introducción, revisar las oraciones e ideas fuerza; también revisar en la introducción si está bien planteado y ubicado el problema; los métodos y procedimientos son los adecuados; son suficientes para convencer al lector de cómo se obtuvieron los resultados, comparar estos con las preguntas planteadas, revisar su presentación en tablas y figuras buscando que se interpreten por si solas. Examinar si la discusión de resultados empieza por la hipótesis, si son explicados en forma adecuada los alcances de los resultados. Analizar si el documento cierra en forma adecuada y termina con un final apropiado. Si se resalta la significancia de los resultados. Reflexionar el artículo como si se fuera un lector o un evaluador.

\section{Proceso de publicación}

\section{Selección de la revista}

Cuando ya se tiene el trabajo muy bien revisado y se cuenta con la seguridad de que puede ser publicado, es necesario seleccionar la revista a la cual se enviará. La mejor práctica es seleccionarla desde la escritura del artículo, con la finalidad de adecuar las diferentes partes a las características y estructura de la publicación. Para una buena selección es conveniente atender algunos criterios: la revista mantiene un proceso de revisión por pares, está soportada por una editorial o una institución acreditada, cuenta con buena reputación en la comunidad científica, posee un editor o editora responsable y con el respaldo de editores temáticos; el tiempo en el cual ha sido publicada sin interrupciones, publicada a tiempo respetando la cantidad de veces que establece en el año, tiene cuidado con las revistas que se encuentran en etapa de reconfiguración o etapa de transición. Un elemento muy importante por atender es el número de índices de calidad con que cuenta la revista, pues esta constituye una buena posibilidad de incrementar su visibilidad, así como también conocer con qué factor de impacto cuenta la revista o simplemente llevar a cabo una exploración para saber con qué tipo de lectores cuenta.

\section{Carta de presentación}

Una atención que debe tener el autor o autora es acompañar el artículo con una carta de presentación que explique el contenido del trabajo, las razones por las que se selecciona esa revista, resaltar las bondades de esta y mencionar si algún investigador en especial la ha recomendado. 
URL: http://www.una.ac.cr/educare

CORREO: educare@una.cr

Belcher (2009) propone que la carta atienda los siguientes puntos: nombre del editor y saludo cordial, mencionar algunas conexiones que ha tenido con la revista, anotar por qué el editor y la revista deben estar interesados en la publicación del trabajo, mostrar conocimiento de la revista, mencionar el título del trabajo e incluir un resumen, resaltar que el artículo es original y no se ha mandado a otra publicación, establecer la disposición para atender las correcciones que sugieran los árbitros y, por último, dar las gracias al editor por las atenciones y por la oportunidad que se le brinda al trabajo para ser evaluado.

\section{Esperando los resultados}

Cuando llega el artículo a la revista, la primera actividad del editor o editora es revisar si el tema es pertinente a la problemática que atiende la revista, después si las instrucciones a los autores fueron atendidas debidamente. Si se cumplen estos requisitos, el editor transfiere el artículo a los evaluadores que generalmente son dos. Los resultados pueden ser: aceptado sin observaciones, cuando los dos evaluadores aceptan el artículo y no realizan ninguna recomendación, evento que es muy poco probable; aceptado con observaciones, donde cada evaluador realiza las observaciones, las envía al editor y este al autor, quien tiene la libertad de hacer las correcciones o desatender y no publicar el artículo. También puede presentarse el caso de que un evaluador acepte y el otro rechace, entonces la decisión la toma un tercer evaluador. Otra opción es el rechazo, en este caso lo único que se puede hacer es reformular el trabajo, adecuarlo a las características de otra revista y enviarlo de nuevo, jpero de ya!, como dice S. Álvarez (comunicación personal, 19 de junio, 2012).

Disponiendo de la decisión de los evaluadores, es necesario planear la repuesta y atender en forma adecuada las observaciones.

Si la decisión es el rechazo, se hace necesario revisar las posibles razones, las cuales pueden venir de la forma de abordar la temática en cuestión -puede ser demasiado amplia o muy restringida, de tal manera de que no convenza a los árbitros-; es un trabajo fuera de tema y de interés de la revista -esto la va a decidir el editor-; es un artículo no académico, es decir, no tiene la estructura ni el tratamiento científico que se le debe dar a un tema; se sitúa demasiado a la defensiva, esto es, escribe utilizando varias disculpas, de porqué no se tratan determinadas cuestiones; carencia de originalidad en el tratamiento de la problemática planteada; manifiesta una pobre estructura alejada de un artículo científico; cuenta con demasiadas fallas teóricas y metodológicas; la escritura y la ortografía no corresponden a un investigador científico. Leach (2002), basado en su experiencia como editor de una revista, señala que la principal razón de rechazo es que el autor yerra al probar la hipótesis propuesta; además, porque el trabajo no agrega elementos al avance del conocimiento sobre el tema, o quizá el tema ya se ha publicado por otros autores con los mismos resultados; o bien se hace un uso incorrecto de los datos. 
En las líneas siguientes se exponen, al respecto, opiniones de los investigadores:

E. Fernández (comunicación personal, 22 de junio, 2012) plantea que un artículo puede ser rechazado por falta de precisión técnica; por carencia de originalidad, por incoherencia en la exposición; porque la construcción y abordaje del objeto de estudio nos son los adecuados, no cuenta con conclusiones significativas que se deriven de forma lógica del cuerpo del trabajo; por último, el lenguaje y la redacción no son los propicios.

Ò. Contreras (comunicación personal, 29 de junio, 2012) señala que esto depende del tipo de revista, las exigencias con las que la publicación trabaja, pero considerando las más profesionales, las principales causas son: falta de rigor académico; carencia de originalidad; desconocimiento de la bibliografía especializada del tema, lo cual suele reflejarse en un mal planteamiento del problema y en un análisis poco relevante, y la no correspondencia del artículo con la política editorial de la revista.

Por su parte, V. Corral (comunicación personal, 26 de junio, 2012) establece, como motivo de rechazo, la falta de originalidad (escribir sobre réplicas de modelos ya probados), empleo de los propios estudiantes como sujetos de investigación (esto es en psicología), mala redacción, falta de claridad en la exposición, abuso del discurso en detrimento de la exposición de datos.

S. Álvarez (comunicación personal, 19 de junio, 2012) señala que la revista no es la apropiada para el tema de la contribución. La contribución es muy horizontal (más de lo mismo) y no aporta material nuevo del tipo que empuja la frontera del conocimiento. Hay fallas graves en los supuestos o en los datos. El procesamiento de los datos es inapropiado (no hay pruebas estadísticas robustas).

E. Méndez (comunicación personal, 14 de junio, 2012) señala: redacción confusa, irrelevancia del problema, inconsistencias.

\section{Restricciones y recomendaciones}

Esta sección del trabajo se desarrolló, principalmente, con base en las entrevistas con los investigadores.

\section{Limitantes}

A este respecto, Belcher (2009) marca veintiocho obstáculos al proceso de escritura y publicación de un artículo científico, aquí se recogen en forma resumida estos obstáculos: escasez de tiempo, la dedicación a la preparación e impartición de clases, posponer la escritura por diversos motivos, no disponer de un adecuado estado de ánimo, no contar con un espacio físico adecuado, falta de lecturas apropiadas, disponer de ideas muy controvertidas, temor a la publicación, brindar atención a la familia, carecer de concentración para escribir por tanto las 
URL: http://www.una.ac.cr/educare

CORREO: educare@una.cr

ideas se van, no contar con asesores adecuados, escribir y trabajar muy despacio, realizar varias actividades al mismo tiempo, necesidad de mayor inteligencia, dificultad para la escritura, por último, se argumenta que el tiempo se va en contestar correos, llenar formatos y escribir reportes.

En palabras de los investigadores entrevistados las limitantes para la escritura de artículos científicos se presentan de la forma siguiente:

Falta de familiaridad con las "convenciones" del formato de artículo científico; temor a exponerse ante la crítica severa (muchas veces este temor se oculta detrás del argumento de que el arbitraje no es imparcial, o que solo le publican a autores ya consolidados, o que las revistas extranjeras no se interesan por los problemas de "nuestro país"); desaliento ante los dictámenes negativos; falta de un entorno académico donde el artículo científico sea valorado, y creciente facilidad para publicar en compilaciones, memorias y "libros de ocasión" que les reditúan "puntos" en las evaluaciones académicas (O. Contreras, comunicación personal, 29 de junio, 2012).

A. Hualde (comunicación personal, 25 de junio, 2012) se refiere principalmente al desconocimiento de las revistas, o sea, el artículo no se envía a la revista adecuada. En este aspecto la revista seleccionada puede tener diferente enfoque disciplinario al trabajo en cuestión, también puede ser que se envíe a una revista internacional con datos meramente locales, por la carencia de un encuadre de espacio adecuado. Se puede presentar el caso de que, aunque el arbitraje es anónimo, la revista o los árbitros no consideren al autor dentro del grupo científico "selecto".

S. Álvarez (comunicación personal, 19 de junio, 2012), por su parte, señala que la falta de experiencia, falta de dominio del idioma inglés (aún si estudió en un país de habla inglesa) y el desconocimiento de las revistas más apropiadas al tema en cuestión constituyen los principales obstáculos.

Corral (comunicación personal, 26 de junio, 2012) insiste en la poca experiencia del autor en estos procesos y que se haya formado en un programa de doctorado con bajo nivel académico.

E. Fernández (comunicación personal, 22 de junio, 2012) apunta la dificultad para expresarse en un idioma extranjero; limitaciones para formalizar sus ideas, para estructurar adecuadamente el trabajo, cuando se empieza, es más difícil, puede ser muy duro penetrar la coraza del arbitraje riguroso, después de que el autor empiezas a ser conocido las cosas se simplifican. Este fenómeno es aún más agudo cuando el joven es latino, africano o asiático.

E. Méndez (comunicación personal, 14 de junio, 2012) menciona el desconocimiento del trabajo por parte de los pares que lo evaluarán; falta de entrenamiento en el manejo de las formas y requisitos de presentación. Lo que señala este investigador ocurre si la revista no selecciona los pares adecuados, lo cual es un hecho posible -aunque remoto, puede presentarse-. 


\section{Recomendaciones}

Ante las anteriores limitaciones, los investigadores formulan las recomendaciones siguientes:

Las sugerencias de S. Álvarez (comunicación personal, 19 de junio, 2012) resultan muy acertadas, en primer lugar, si quiere ser investigador debe leer bastante, ya que escribe bien quien lee mucho. Un segundo elemento es que sea osado, que se atreva. Que no se detenga porque "no posee suficientes datos". Que tenga conciencia de que si se le piden correcciones ya está del lado positivo del negocio, y que si se le rechaza su trabajo puede hacer correcciones y enviarlo a otra revista, pero de forma inmediata.

A. Hualde (comunicación personal, 25 de junio, 2012) sugiere que se debe adecuar el texto al tipo de revista seleccionada, cuidar la metodología, vigilar la coherencia interna, tener en cuenta que las referencias estén actualizadas. Es necesario leer artículos antes de publicar para que ver la forma cómo se encuentran estructurados. Menciona que a riesgo de parecer malinchista, opina que las revistas en inglés son en general mucho más exigentes y se aprende más a partir de su lectura. También se aprende enviando artículos a dictamen de revistas en inglés, pues los filtros son muy exigentes.

O. Contreras (comunicación personal, 29 de junio, 2012) señala que los académicos interesados en publicar deben leer artículos científicos en las revistas más importantes de su tema/disciplina, para familiarizarse con las convenciones del género. Cuidar al máximo que el problema planteado en el artículo sea relevante desde el punto de vista de los debates importantes para la comunidad académica ocupada de este tipo de temas/problemas.

Proponer problemas de alcance modesto, pero con el mayor rigor y claridad posibles.

Especializarse al máximo, por lo menos durante un número razonable de años, para lograr incidir en los debates de un tema o problema, antes de pensar en abarcar otros temas o cambiar de área de especialización. Esto implica publicar varios artículos científicos sobre el mismo tema/problema.

Corral (comunicación personal, 26 de junio, 2012) opina que se debe trabajar mucho, adquirir práctica, escribir con investigadores más experimentados, enviar a revisión de expertos sus artículos antes de someterlos a dictamen.

E. Fernández (comunicación personal, 22 de junio, 2012) recomienda corregir el idioma por un experto o por un nativo en caso de lenguaje extranjero, siempre que se lea un artículo observar lo que es común en su estructura. Atenerse a ella al escribir; pensar siempre que el árbitro va a pedir la evidencia más convincente sobre la calidad de lo que se propone. Cuando no se está seguro de que un trabajo sea suficientemente consistente o esté suficientemente completo, es preferible no enviarlo. Se deben realizar correcciones hasta obtener completa satisfacción. Resulta más provechoso, a la larga, un artículo bueno que dos apenas aceptables. Evitar escribir artículos con mejoras marginales (el segundo respecto al primero). Si es una buena revista, los árbitros no dejan pasar semejantes contribuciones incrementales. 
URL: http://www.una.ac.cr/educare

CORREO: educare@una.cr

E. Méndez (comunicación personal, 14 de junio, 2012) dice que es necesario evitar la paráfrasis en forma exagerada y la perífrasis mal elaborada; ubicarse explícitamente en el ámbito de producción sobre el problema a tratar; hacer legible la estructura y estrategia narrativa.

En la actualidad, una de las formas más comunes de plagio es a través de parafrasear en forma inadecuada faltando a los principios de la ética científica. Rojas $(2012$, p. 58) apunta que los principales errores que se cometen al recurrir a una paráfrasis son: "Se parafrasea y no se anota la fuente original. El parafraseo es continuo y extenso, no se añade material que permita interacción o enriquezca la información, a pesar de que se menciona la fuente. Los trabajos académicos -que exigen pensamientos originales y reflexiones críticas sobre los puntos de vista de otras personas-se convierten en textos que no superan la repetición. No se identifican claramente, como tales, los pasajes parafraseados".

\section{Principales vicios y errores}

V. Corral (comunicación personal, 26 de junio, 2012) señala que los principales vicios son: ser repetitivos, escribir de manera monótona, cometer faltas de ortografía, no atender las recomendaciones y críticas de los revisores.

Por su parte, A. Hualde (comunicación personal, 25 de junio, 2012) opina que, un primer vicio es revisar el conocimiento científico producido en otros países sin una visión crítica y sin seleccionar lo que realmente es pertinente para el tema del artículo. Además de eso, le parece necesario insistir en la metodología, se encuentran muchos artículos con una metodología inconsistente. Un tercer factor a tener en cuenta es abrir el artículo con un planteamiento interesante y coherente, distinguir entre la descripción y el análisis; recomienda finalmente recapitular lo que se argumenta en distintos momentos del texto y cuidar mucho las conclusiones. De lo contrario, se incurre en un vicio de incoherencia que distrae al lector y debilita el rigor argumental.

O. Contreras (comunicación personal, 29 de junio, 2012) opina que el investigador supone que el problema que aborda no ha sido investigado antes, lo cual puede ser por falta de conocimiento de los avances en su área de estudio. El artículo presentado no plantea realmente un problema, sino un conjunto de reflexiones temáticas. El investigador supone que una investigación local/regional no tiene conexión con los grandes debates internacionales. El "marco teórico" no corresponde con el análisis de los datos. El trabajo no se propone discutir un "problema científico" sino resolver problemas económicos o sociales y se encierra solo en propuestas descriptivas.

Otro investigador, S. Álvarez (comunicación personal, 19 de junio, 2012), menciona como principales vicios el pulverizar demasiado la aportación en un afán de aumentar el número de artículos publicados. Un error serio es pretender publicar lo mismo en dos revistas (una 
extranjera y otra mexicana): no se valen los refritos. Un error es creer que la audiencia ya sabe todos los símbolos y conceptos: hay que definir los símbolos y clarificar los conceptos (sin abundar demasiado).

E. Fernández (comunicación personal, 22 de junio, 2012) señala las imprecisiones en el contenido, manejo "desaseado" del idioma y escasa formalización, pobre estructuración y no dejar bien claro el valor de la propuesta. Enviar trabajos que en realidad no están razonablemente completos. Poca evidencia de la calidad de la propuesta en comparación con los benchmarks. Intentar hacer dos artículos de lo que pudiera ser uno solo más fuerte. No referenciar las fuentes más actuales. Conclusiones débiles o poco justificadas en el cuerpo del trabajo y, por último, creer ingenuamente que los árbitros son bien intencionados y superficiales.

E. Méndez (comunicación personal, 14 de junio, 2012) establece como principales vicios la omisión de fuentes y aportaciones recientes; falta de fundamentación de la relevancia y pertinencia de la elaboración; adscripción teórico metodológica fuera de contexto.

\section{Experiencia en la evaluación de artículos científicos}

A los entrevistados se les preguntó acerca de su experiencia como evaluadores en las revistas científicas y en qué partes concentran su atención. Las respuestas se expresan a continuación.

E. Fernández (comunicación personal, 22 de junio, 2012) opina que primero es necesario insistir en la precisión técnica, es decir, en los datos y el tratamiento a los mismos, la originalidad del artículo, las ventajas de la propuesta con relación con el estado de la temática en cuestión, la coherencia en la exposición, la estructuración del artículo, conclusiones significativas que sean resultados de la discusión del artículo, todo ello sin desatender el uso del lenguaje y la redacción.

S. Álvarez (comunicación personal, 19 de junio, 2012) insiste en que es necesario fijar la atención en la originalidad de la aportación, en la claridad del escrito y la veracidad de las aseveraciones (esto implica verificar las expresiones matemáticas y los cálculos).

O. Contreras (comunicación personal, 29 de junio, 2012) establece como focos de atención el dominio del estado del conocimiento existente respecto del tema/problema tratado en el artículo. Que el artículo efectivamente formule un problema pertinente (un problema "científico", independientemente de que el trabajo pueda tener valor -o no- para la formulación de políticas o para la solución de problemas sociales). Que el trabajo tenga una adecuada articulación entre la teoría, la metodología y el análisis. Que realice una contribución al conocimiento, aunque sea modesta.

V. Corral (comunicación personal, 26 de junio, 2012) concentra su atención en la originalidad del artículo (que, por lo menos, contribuya con algo diferente al conocimiento 
URL: http://www.una.ac.cr/educare

CORREO: educare@una.cr

actual), que los antecedentes lleven a la propuesta de investigación (relación entre el método y el marco teórico), descripción detallada -pero no tediosa- del método, corroborar que los resultados no incluyan valoraciones de los datos y que en las conclusiones se haga referencia a los datos del artículo.

E. Méndez (comunicación personal, 14 de junio, 2012) revisa principalmente el estado del arte; la pertinencia-relevancia-originalidad; claridad y calidad de redacción; secuencia lógica en la presentación; congruencia entre objetivo-hipótesis-tratamiento-resultado.

\section{Conclusiones}

La habilidad y experiencia en la presentación de reportes de investigación a través de la elaboración y publicación de artículos científicos solo se logra mediante el ejercicio cotidiano. En el presente trabajo únicamente se presentan ideas para organizar de mejor manera este ejercicio, en ningún momento constituye una fórmula prodigiosa que sustituya el trabajo y ejercicio permanentes. Más bien, la presente comunicación pretende ser una invitación a los investigadores jóvenes para para que se comprometan con este tipo de tareas.

En México, las instituciones de educación superior y los centros de investigación científica tienen la responsabilidad central de impulsar los procesos de producción de conocimiento a través de la realización de proyectos de investigación que empujen las fronteras del saber, a la vez, deben fortalecer los procesos de difusión y divulgación de la ciencia. La forma mayormente aceptada por la comunidad académica para la difusión de la ciencia es a través de la publicación de artículos científicos, por lo que en los diferentes programas de posgrado, centrados en la investigación, además de enseñar a investigar, resulta de suma importancia impulsar talleres que lleven a los estudiantes a adquirir práctica en la elaboración y publicación de artículos científicos, estos talleres deben ser requisitos formales para la obtención del grado.

También existe la necesidad de un mayor vínculo entre las comunidades académicas; esto, con la finalidad de fortalecer las redes de colaboración, donde el análisis y discusión de los reportes de resultados - manifiesta en artículos científicos-resulte una forma de acercamiento e integración de las comunidades académicas en nuestro medio. Un artículo científico bien estructurado constituye el centro de la tarea académica y, por tanto, eslabona el quehacer de una comunidad científica.

\section{Agradecimientos}

Por sus aportaciones al presente trabajo, el autor agradece a los siguientes Miembros del Sistema Nacional de Investigadores Nivel III:

- Saúl Álvarez Borrego. Investigador titular del Departamento de Ecología Marina, Centro de Investigación Científica y Educación Superior de Ensenada (CICESE), Ensenada, Baja California, México. 
- Óscar Fernando Contreras Montellano. Investigador del Departamento de Estudios Sociales, Área Industria y Empleo, Colegio de la Frontera Norte, Tijuana, Baja California, México.

- Víctor Corral Verdugo. Investigador de la División de Ciencias Sociales, Departamento de Psicología y Ciencias de la Comunicación, Universidad de Sonora, Hermosillo, Sonora. México.

- Eduardo René Fernández González. Investigador de la Facultad de Ingeniería, Universidad Autónoma de Sinaloa, Culiacán, Sinaloa, México.

- Alfredo Hualde Alfaro. Investigador del Departamento de Estudios Sociales, Área de Sociología del Trabajo, Sistemas Regionales de Innovación y Economía del Conocimiento, Colegio de la Frontera Norte, Tijuana, Baja California, México.

- Eloy Méndez Sainz. Investigador del Centro de Estudios de América del Norte, Colegio de Sonora, Hermosillo, Sonora, México.

\section{Referencias}

American Psychological Association (APA). (2010). Publication Manual of the American Psychological Association [Manual de publicaciones de la American Psychological Association (6 $6^{\mathrm{a}}$ d.). Washington: American Psychological Association.

Baltzopoulos, V. (2003). Writing a scientific paper for publication [Escritura de un artículo científico para su publicación]. Journal of Sports Sciences, 21(6), 441-442. doi: 10.1080/0264041031000101854

Belcher, W. L. (2009). Writing Your Journal Article in 12 Weeks. A Guide to Academic Publishing Success [Escritura de su artículo científico en 12 semanas. Una guía para el éxito de las publicaciones académicas]. Thousand Oaks: Sage.

Booth, V. (2006). Communicating in Science. Writing Scientific Paper and Speaking at Scientific Meetings [La comunicación de la ciencia. Escritura de artículos científicos y discursos en reuniones científicas] ( $2^{\mathrm{a}}$ Ed.). Cambridge: Cambridge University Press.

Campanario, J. M. (2003). Cómo escribir y publicar un artículo científico. Cómo estudiar y aumentar su impacto. Revista Española de Documentación Científica, 26(4), 461-463. doi:10.3989/redc.2003.v26.i4.203

Cargill, M. y O'Connor, P. (2009). Writing Scientific Research Articles. Strategy and Steps [La escritura de artículos de investigación científica. Estrategias y pasos]. West Sussex: WileyBlackwell.

Contreras, O. F. (2011). La comunidad académica y sus medios: La consolidación de una revista de ciencias sociales. Región y Sociedad, 23(50), 11-22. 
URL: http://www.una.ac.cr/educare

CORREO: educare@una.cr

Cortex (septiembre, 2001). Interview to Eugene Garfield, Chairman Emeritus of the Institute for Scientific Information (ISI) [Entrevista a Eugene Garfield, presidente emérito del Instituto para la información Científica] (Sección editorial). Cortex, 37(4), 575-577.

Day, R. A. y Gastel, B. (2006). How to Write and Publish a Scientific Paper [Cómo escribir y publicar un artículo científico] (6 $6^{\mathrm{a}}$ Ed.). London: Greenwood Press.

Garfield, E. (2003). The Meaning of the Impact Factor [El significado del factor de impacto]. Revista Internacional de Psicología Clínica y de la Salud, 3(2), 363-369. Recuperado de http://garfield.library.upenn.edu/papers/meaningofif2003.pdf

Garson, D. G. (2002). Guide to Writing Empirical Papers, Theses, and Dissertations [Guía para redactar trabajos empíricos, tesis y tesinas]. New York: Marcel Dekker.

González, Y.y Jiménez, J. (2012). La articulación, requisito básico de un producto de investigación educativa. Revista Electrónica Educare, 16(1), 5-13. Recuperado de http://www.revistas. una.ac.cr/index.php/EDUCARE/article/view/3728

Körner, A. M. (2004). Guide to Publishing a Scientific Paper Paper [Guía para publicar un artículo cientifico]. New York: Routledege.

Leach, R. E. (Septiembre, 2002). How to Write a Scientific Paper [Cómo escribir un artículo científico]. Sports Medicine and Arthroscopy Review, 10(3), 228-233.

Licea, J. y Santillán-Rivero, E. G. (enero-junio, 2002). Bibliometría ¿Para qué? Biblioteca Universitaria, 5(1), 3-10. Recuperado de http://www.dgbiblio.unam.mx/servicios/dgb/ publicdgb/bole/fulltext/volV12002/pgs-03-10.pdf

Lodeiros-Seijo, C., De Donato, M. y Monge-Nájera, J. (2002). Manual práctico de redacción y crítica de artículos científicos. Cumaná: Universidad de Oriente.

López-Leyva, S., Alvarado-Borrego, A. y Mungaray-Moctezuma, A. B. (2011). La comunicación de la ciencia a través de artículos científicos. México: Juan Pablos Editores y U de O.

Mantilla-Villarreal, A, Medina-Fontalvo, J., Velasco-Bayuelo, C. A., Algarín-Gregory, J., RodeloSalcedo, E., De la Rosa-Barranco, D. y Caballero-Uribe, C.. V. (julio-diciembre, 2010), "Guía práctica para publicar un artículo en revistas latinoamericana", Salud Uninorte, 26(2), 311-324. Recuperado de http://redalyc.uaemex.mx/src/inicio/ArtPdfRed. jsp?iCve $=81719006012$

Mari-Mutt, J. A. (2010). Manual de redacción científica. Recuperado de http://edicionesdigitales. info/Manual/Manual/invypubl.html

Quispe, C. (2004). ¿Es el factor de impacto un buen indicador para medir la calidad de las revistas científicas?: Análisis de algunos problemas generados por su uso. Infobib, 3, 1-19. Recuperado de http://eprints.rclis.org/5002/1/articulo1.pdf 
Rogel, R. (2009). Apuntes del curso: "Elaboración de artículos científicos y criterios de arbitraje académico", impartido por la Asociación Nacional de Instituciones de Educación Superior, ciudad de México, 9-11 de febrero de 2009.

Rojas, M. E. (2012). Plagio en textos académicos. Revista Electrónica Educare, 16(2), 55-66. Recuperado de http://www.revistas.una.ac.cr/index.php/EDUCARE/article/view/3930

Sabaj, O. (2009). Descubriendo algunos problemas en la redacción de artículos de investigación científica (AIC) de los alumnos de postgrado. Revista Signos, 42(69), 107-127. Recuperado de http://www.scielo.cl/pdf/signos/v42n69/a06.pdf

Sabaj, O. y Páez, D. (2010). Tipos y funciones de las citas en artículos de investigación de tres disciplinas. Literatura y Lingüística, 22, 117-134. Recuperado de http://www.scielo.cl/pdf/ lyl/n22/art09.pdf

Sharp, D. (junio, 2002). Kipling's Guide to Writing a Scientific Paper [Guía de Kipling para escribir un artículo científico]. Croatian Medical Journal, 43(3), 262-267. Recuperado de http:// neuron.mefst.hr/docs/CMJ/issues/2002/43/3/12035130.pdf

\section{Cómo citar este artículo en APA:}

López, S. (2013). El proceso de escritura y publicación de un artículo científico. Revista

Electrónica Educare, 17(1), 5-27. Recuperado de http://www.revistas.una.ac.cr/index. php/EDUCARE/issue/current

Nota: Para citar este artículo en otros sistemas puede consultar el hipervínculo "Como citar el artículo" en la barra derecha de nuestro sitio web:

http://www.revistas.una.ac.cr/index.php/EDUCARE/index 